\title{
Association between MIC-1 and Type 2 Diabetes: A Combined Analysis
}

\author{
Jianan Lu $\mathbb{D}$, , Yue Zhang $\mathbb{D}^{1},{ }^{1}$ Xingxuan Dong $\mathbb{D}^{1},{ }^{1}$ Jiawen Lu $\mathbb{D}^{1},{ }^{1}$ Chen Zhang ${ }^{(D)},{ }^{1}$ Jieyu Liu $(\mathbb{D})$, \\ Qingzhou Yu $\mathbb{D},{ }^{1}$ Haoyue Teng $\mathbb{D}^{1},{ }^{1}$ Qingkui Yao $\mathbb{D}^{\circ},{ }^{2}$ Jieyun Yin $\mathbb{D}^{1},{ }^{1}$ and Liqiang Qin $\mathbb{D}^{3}$
}

\author{
${ }^{1}$ Department of Epidemiology and Biostatics, Jiangsu Key Laboratory of Preventive and Translational Medicine for \\ Geriatric Diseases, School of Public Health, Medical College of Soochow University, 199 Ren Ai Road, Suzhou, China \\ ${ }^{2}$ Community Health Service Center of Minglou, Subdistrict Jiangdong District, Ningbo, China \\ ${ }^{3}$ Department of Nutrition and Food Hygiene, School of Public Health, Soochow University, Suzhou, China
}

Correspondence should be addressed to Jieyun Yin; jyyin@suda.edu.cn

Received 19 April 2019; Revised 23 September 2019; Accepted 3 October 2019; Published 16 November 2019

Academic Editor: Ralf Lichtinghagen

Copyright ( 2019 Jianan Lu et al. This is an open access article distributed under the Creative Commons Attribution License, which permits unrestricted use, distribution, and reproduction in any medium, provided the original work is properly cited.

Background and Objectives. Type 2 diabetes mellitus (T2DM) is an epidemic disease that endangers human health seriously. Recently, a large number of reports have revealed that macrophage-inhibiting cytokine-1 (MIC-1) is linked with T2DM, but the results were inconclusive. The aim of this study was to perform bioinformatics analysis of the association between MIC-1 and T2DM. Material and Methods. Datasets and relevant literatures were searched in Gene Expression Omnibus (GEO), PubMed, Google Scholar, and Web of Science till September 20, 2019. Expression levels of MIC-1 were extracted, pooled, and compared between T2DM cases and controls. Results. In summary, 11 GEO datasets and 3 articles with 421 T2DM cases and 711 controls were finally included. The expression level of MIC-1 was significantly higher in T2DM patients compared with controls, with a standard mean difference (SMD) of 0.54 and a $95 \%$ confidence interval (95\% CI) of $0.24-0.83$; in blood samples, the difference was still significant $(\mathrm{SMD}=0.65 ; 95 \% \mathrm{CI}=0.24-1.06)$. Meanwhile, the expression level of $M I C-1$ plays a significant role in differentiating T2DM cases from controls; the combined sensitivity, specificity, and odds ratio were $0.83(95 \% \mathrm{CI}=0.72-0.90)$, $0.59(95 \% \mathrm{CI}=0.45-0.72)$, and $1.64(95 \% \mathrm{CI}=1.35-1.99)$, respectively. The summary receiver operating characteristic (SROC) curve demonstrated that the area under the curve (AUC) was $0.81(95 \% \mathrm{CI}=0.77-0.84)$. Conclusion. Our results suggested that the expression levels of MIC-1 were significantly higher in T2DM patients in multiple tissues including blood samples.

\section{Introduction}

Diabetes is a global disease which is described as a type of metabolic disorder distinguished by increased blood glucose concentration. At present, nearly half a billion people suffer from diabetes [1], and the number of people affected with diabetes has been rising for the last decades [2]. Type 2 diabetes mellitus (T2DM) is the most common type of diabetes and accounts for around $90 \%$ of total diabetes cases [3]. Overweight and obesity have been regarded as the main risk factors contributing to T2DM [4]. Approximately 50\% of obese subjects will develop into T2DM [5]. Thus, pathway targeting energy metabolism may provide useful diagnosis and treatment information for T2DM [6].
Macrophage-inhibiting cytokine-1, also termed growth differentiation factor 15 (GDF-15), encodes a secreted protein of transforming growth factor beta (TGF-beta) family [7]. It is weakly expressed in multiple tissues under normal conditions [7]. The upregulated production of MIC-1 is triggered in response to inflammation such as tissue injury, biomechanical stress, and anoxia $[8,9]$. MIC-1 has been considered to play a pivotal role in the development and progression of many diseases such as cardiovascular diseases and malignant cancer [10-12]. Recently, MIC-1 is considered as a long-term metabolism regulator with a function of increasing lipolysis as an adipokine in a paracrine fashion [13-15]. There have been evident findings in mice that overexpression of MIC-1 could lower the preference 
for fat intake and improve glucose tolerance and insulin sensitivity compared with MIC-1-null mice which further manifests its protective role in energy homeostasis [16-18].

Although several studies have depicted the specific role of MIC-1 in energy expenditure and metabolic activities [13, 14, 19-22], current findings clarifying the relationship between MIC-1 and T2DM are still rare. In our study, we conducted a meta-analysis based on a combination of GEO datasets and relevant clinical reports to illustrate the association between MIC-1 expression levels and T2DM.

\section{Materials and Methods}

2.1. Data Acquisition and Search Strategy. A well-established database of microarray was searched for the current metaanalysis up to September 20, 2019: the Gene Expression Omnibus (GEO; https://www.ncbi.nlm.nih.gov/geo/). The search strategy was as follows: ("diabetes mellitus" OR "diabetes insipidus" OR DIABETES) AND "Homo sapiens".

Afterwards, a systematic literature was searched in PubMed, Google Scholar, and Web of Science, using the combination of key words "diabetes" AND "MIC-1". The language of relevant articles was restricted to English. In order to find more qualified literatures, we viewed the reference lists of publications selected for inclusion.

The process on how we conducted our data search and acquisition of the statistics was described in Figure 1.

2.2. Inclusion and Exclusion Criteria. The inclusion criteria of datasets and suitable literature were as follows: (1) the expression levels of MIC-1 were compared between T2DM patients and nondiabetes people; (2) each study should have at least 10 samples; (3) the original expression profiling data of MIC-1 and its mean and standard deviation (SD) should be offered or could be calculated; (4) samples should be collected from humans.

Studies were excluded if (1) studies have examined other types of diabetes such as type 1 diabetes; (2) studies or data are about animals or cell line; and (3) samples have overlapped with other studies.

2.3. Quality Control and Data Extraction. Two authors (Yue Zhang and Jianan Lu) extracted the information from all qualified datasets in line with the inclusion criteria independently, and any problem or ambiguity was discussed with the team. The following information was extracted for every dataset: last name of first author, country of origin, published year, study subjects (disease status and sample type), expression values, means, and SD of MIC-1.

2.4. Statistical Analysis. At first, we extracted MIC-1 expression levels from datasets and qualified literature; mean and SD were calculated. Next, we performed a meta-analysis to describe expression difference. Forest plots were used to get pooled standard mean difference (SMD) and 95\% confidence interval (95\% CI). We tested the possibility of heterogeneity by Cochran's $Q$-statistic and $I^{2}$ statistics. A $P$ value $<0.05$ and an $I^{2}>50 \%$ were considered as heterogeneous, and the random effect model would be chosen to calculate the pooled SMD [23]. Otherwise, the fixed effect model would be selected. Besides, funnel plots and Begg's test were used to check the potential publication bias. Obesity is usually classified by body mass index: BMI $\geq 30$ as obesity and $\mathrm{BMI}<30$ as nonobesity [24]. As the original data from the literatures were not available, we only included $M I C-1$ expression data from GEO datasets and then conducted diagnostic analysis to assess the diagnostic possibility of $M I C-1$ in T2DM patients. We carried out a meta-analysis with SROC to check the expression level of $M I C-1$ in T2DM patients. Additionally, multivariate analysis of covariance (MANCOVA) was used to test differences in $M I C-1$ between T2DM and non-T2DM groups with age and BMI as covariates in qualified dataset. A nominal level of significance $P<0.05$ was accepted.

The data analyses were performed by SAS (SAS Institute Inc., NC, USA) and STATA (STATA Corporation, College Station, TX, USA).

\section{Results}

3.1. Characteristics of Included Studies. After initial searching from titles and content of abstract, 11 related datasets (GSE9006, GSE20966, GSE12643, GSE13760, GSE16415, GSE23343, GSE25724, GSE38642, GSE26168, GSE19420, and GSE27951) as well as 3 articles were obtained. Finally, a total of 14 studies including 421 T2DM patients and 711 nondiabetes samples were included for the current metaanalysis (Table 1).

3.2. Meta-Analysis of MIC-1 Expression in T2DM Patients and Controls. It was found that the expression of MIC-1 was significantly increased in T2DM patients compared with controls, with SMD of 0.54 (95\%CI = 0.24-0.83) (Figure 2). Additionally, expression levels of MIC-1 in the blood were obviously higher with SMD of $0.65(95 \% \mathrm{CI}=0.24-1.06)$ (Figure 3$)$. There is no publication bias $(P=0.511)$, and the result remained stable according to the sensitivity analysis (Supplementary Figure 1 and Supplementary Figure 2). Subsequently, a diagnostic test was performed to evaluate the diagnostic effect of $M I C-1$ for T2DM patients. Figures 4 and 5 showed the combined sensitivity, specificity, and odds ratio, the corresponding values were $0.83(95 \% \mathrm{CI}=0.72-0.90), 0.59 \quad(95 \% \mathrm{CI}=0.45-0.72)$, and $1.64(95 \% \mathrm{CI}=1.35-1.99)$, respectively. The SROC curve (Figure 6) demonstrated that the area under the curve (AUC) was $0.81(95 \% \mathrm{CI}=0.77-0.84)$.

3.3. MIC-1 and BMI in T2DM. It is of great value to study cardiovascular risk factors in T2DM. Among the included 11 GEO datasets, there were 5 datasets (GSE38642, GSE25724, GSE19420, GSE20966, and GSE27951) with 45 T2DM patients and 95 controls reported information about age and BMI. By utilizing MANCOVA and controlling for age and $\mathrm{BMI}$, the corresponding least-square means of MIC-1 in T2DM patients and controls were shown in Supplementary Table 1. However, the combined difference of least-square means of $M I C-1$ between T2DM and normal controls was not significant in the random model with a 


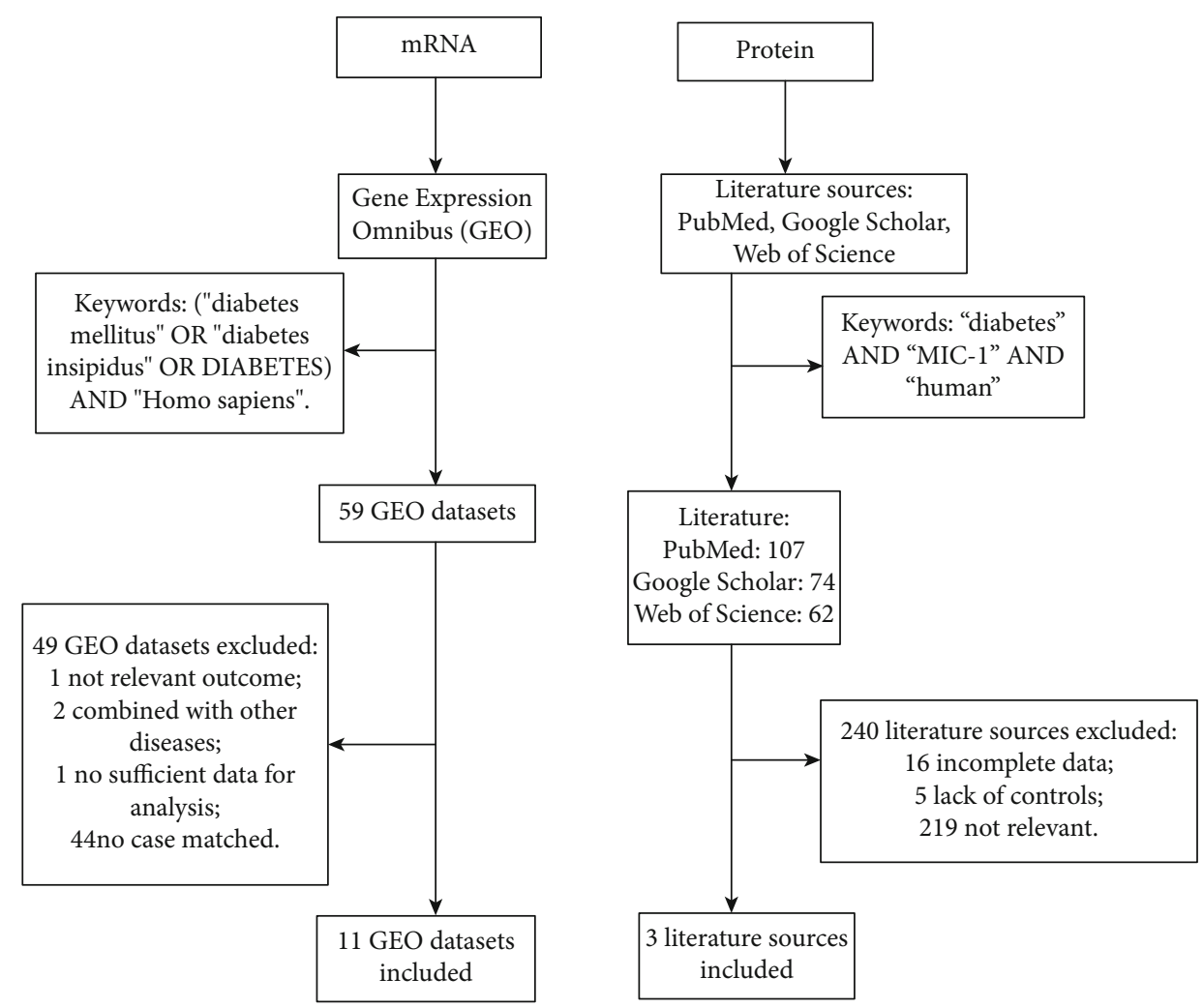

FIGURE 1: Search flow diagram for literature selection.

TABLE 1: Characteristics of MIC-1 expression profiling datasets included in the current meta-analysis between T2DM and non-T2DM population.

\begin{tabular}{|c|c|c|c|c|c|c|c|c|}
\hline Dataset & $\begin{array}{c}\text { Country and } \\
\text { publication year }\end{array}$ & Sample type & Platform & $\begin{array}{c}\text { Tested } \\
\text { substance }\end{array}$ & $\begin{array}{l}\text { Sample } \\
\text { size }\end{array}$ & $\begin{array}{l}\text { Case } \\
\text { MIC-1 mean } \\
\text { and SD }\end{array}$ & $\begin{array}{l}\text { Sample } \\
\text { size }\end{array}$ & $\begin{array}{l}\text { Control } \\
\text { MIC-1 mean } \\
\text { and SD }\end{array}$ \\
\hline GSE20966 & USA, 2010 & Pancreatic beta cell & GPL1352 & mRNA & 10 & $5.647 \pm 3.367$ & 10 & $2.172 \pm 1.655$ \\
\hline GSE12643 & Denmark, 2008 & Myotubes & GPL8300 & mRNA & 10 & $10.345 \pm 0.229$ & 10 & $10.348 \pm 0.408$ \\
\hline GSE13760 & Denmark, 2011 & $\begin{array}{l}\text { Arterial tissue } \\
\text { (intima media) }\end{array}$ & GPL571 & mRNA & 10 & $101.222 \pm 9.536$ & 11 & $95.791 \pm 7.878$ \\
\hline GSE16415 & India, 2009 & $\begin{array}{l}\text { Omentum (visceral } \\
\text { tissue) tissue }\end{array}$ & GPL2986 & mRNA & 5 & $0.736 \pm 0.521$ & 5 & $0.136 \pm 0.098$ \\
\hline GSE23343 & Japan, 2010 & Liver & GPL570 & mRNA & 10 & $0.350 \pm 0.118$ & 7 & $0.438 \pm 0.240$ \\
\hline GSE25724 & Italy, 2010 & Pancreatic islets & GPL96 & mRNA & 6 & $4.660 \pm 0.182$ & 7 & $4.363 \pm 0.242$ \\
\hline GSE38642 & Sweden, 2012 & Pancreatic islets & GPL6244 & mRNA & 9 & $10.000 \pm 0.512$ & 54 & $9.691 \pm 0.626$ \\
\hline GSE19420 & Netherlands, 2010 & $\begin{array}{l}\text { Skeletal muscle } \\
\text { biopsies }\end{array}$ & GPL570 & mRNA & 10 & $3.424 \pm 0.194$ & 12 & $3.632 \pm 0.416$ \\
\hline GSE26168 & Singapore, 2010 & Blood & GPL6883 & mRNA & 9 & $8.278 \pm 7.719$ & 8 & $-3.150 \pm 12.954$ \\
\hline GSE9006 & USA, 2007 & Blood & GPL96 & mRNA & 12 & $19.225 \pm 17.956$ & 24 & $15.308 \pm 13.606$ \\
\hline GSE27951 & UK, 2011 & Adipose tissue & GPL570 & mRNA & 10 & $4.542 \pm 0.271$ & 12 & $4.403 \pm 0.343$ \\
\hline $\begin{array}{l}\text { Castensen } \\
{[41]}\end{array}$ & Germany, 2010 & Blood & & Protein & 180 & $537.100 \pm 166.440$ & 372 & $499.700 \pm 149.330$ \\
\hline $\begin{array}{l}\text { Hong et al. } \\
\text { [30] }\end{array}$ & Korea, 2014 & Blood & & Protein & 75 & $866.040 \pm 628.210$ & 137 & $484.050 \pm 291.000$ \\
\hline $\begin{array}{l}\text { Shin et al. } \\
{[34]}\end{array}$ & Korea, 2016 & Blood & & Protein & 65 & $643.290 \pm 535.390$ & 42 & $210.120 \pm 211.810$ \\
\hline
\end{tabular}




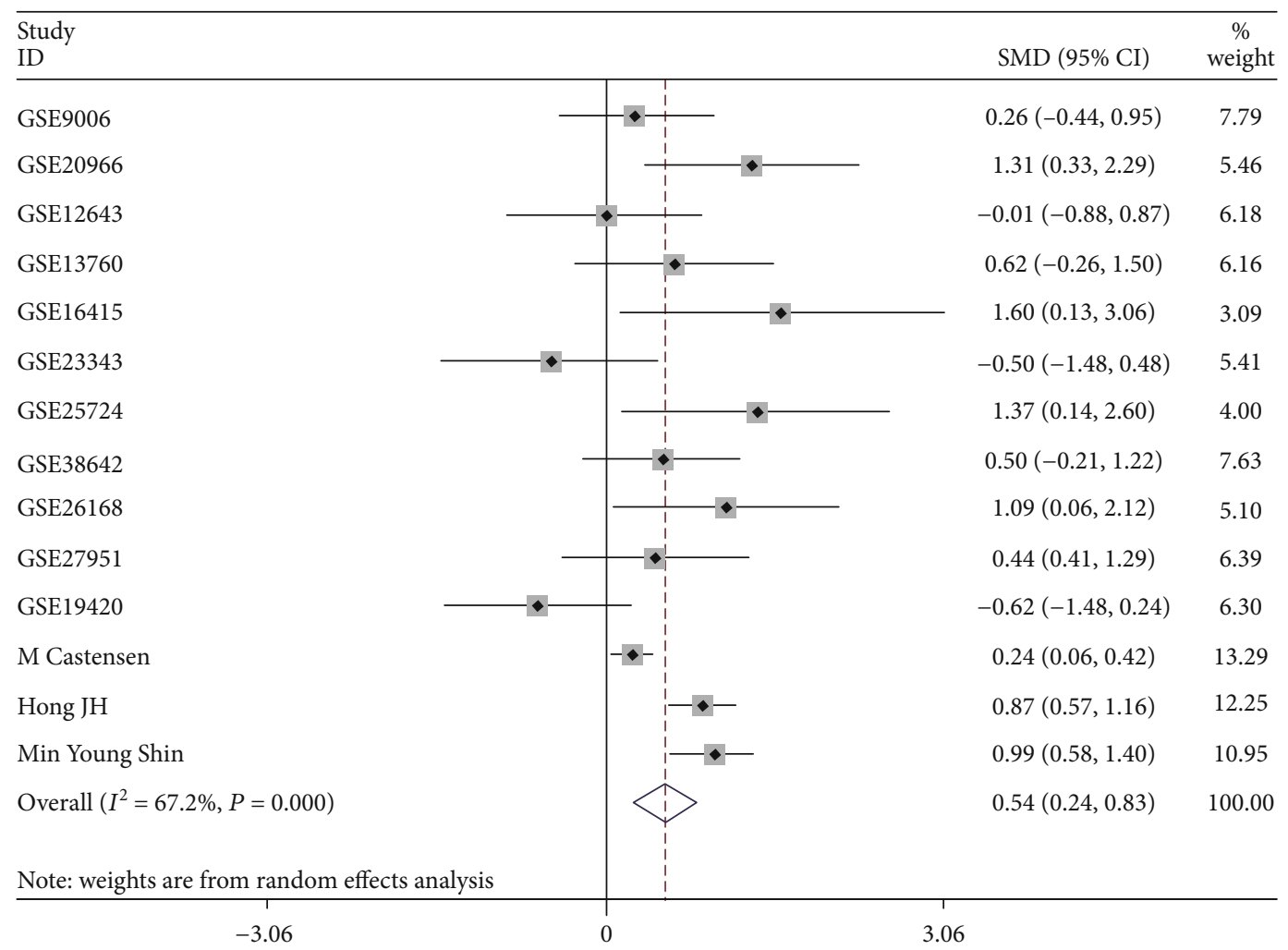

FIGURE 2: Forest plot showing SMD of MIC-1 expression between T2DM patients and nondiabetes people. The random effect model was used in all groups.

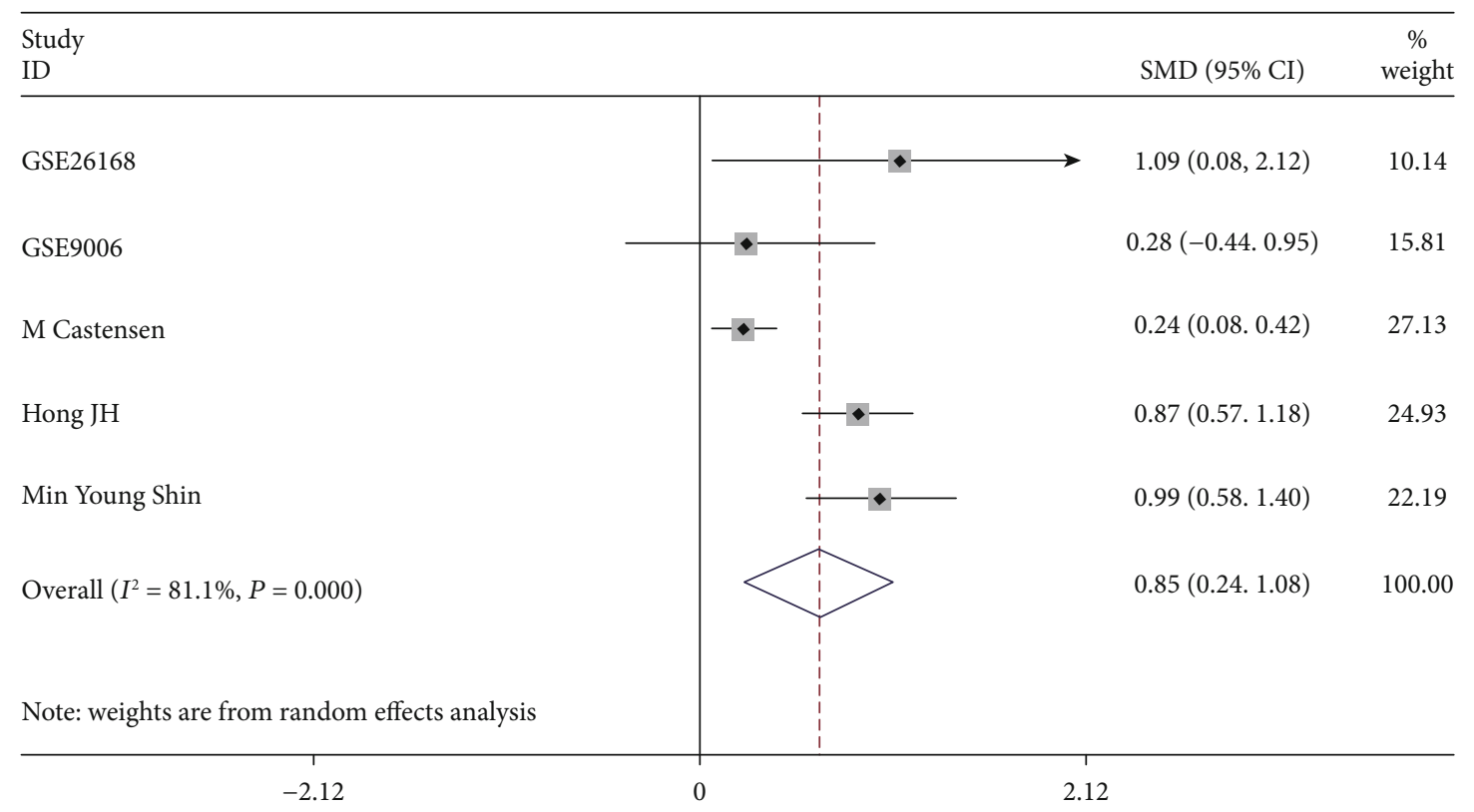

FIGURE 3: Forest plot showing SMD of MIC-1 expression in blood samples between T2DM patients and nondiabetes people. The random effect model was used in all groups.

SMD of $0.78 \quad(95 \% \mathrm{CI}=-0.97-2.53) \quad$ (Supplementary Figure 3).

We also stratified T2DM patients into two groups based on $\mathrm{BMI}$ (BMI $\geq 30$ : obese; $\mathrm{BMI}<30$ : nonobese) in 4 qualified datasets (GSE38642, GSE19420, GSE20966, and GSE27951), which include 25 obese and 21 nonobese T2DM patients (Supplementary Table 2). Compared with T2DM patient that are normal or overweight, obese T2DM patients 


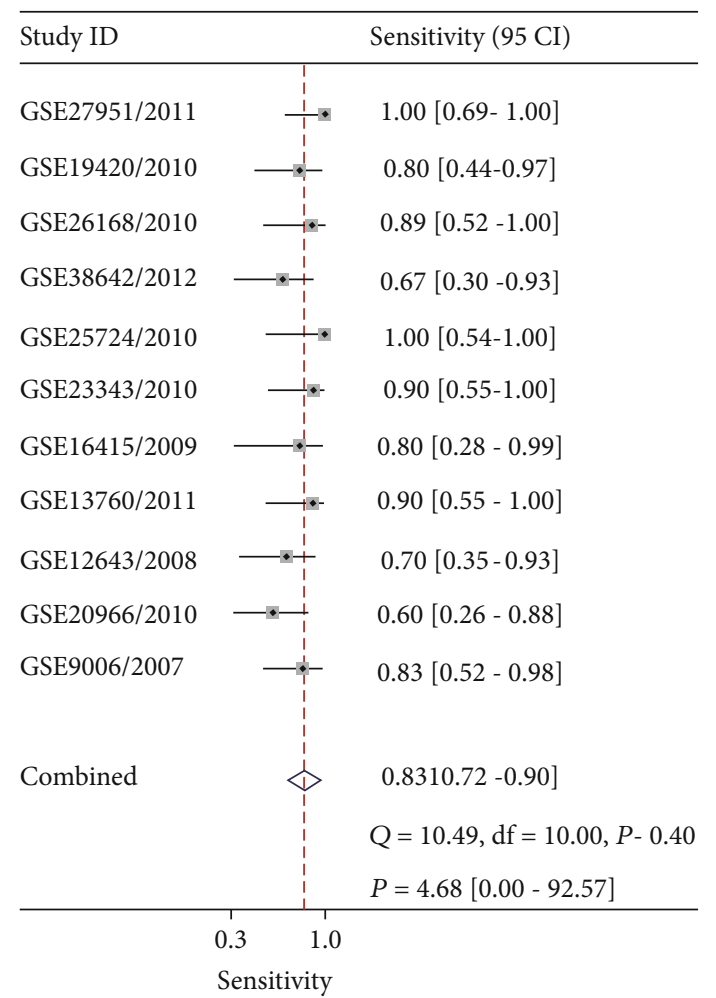

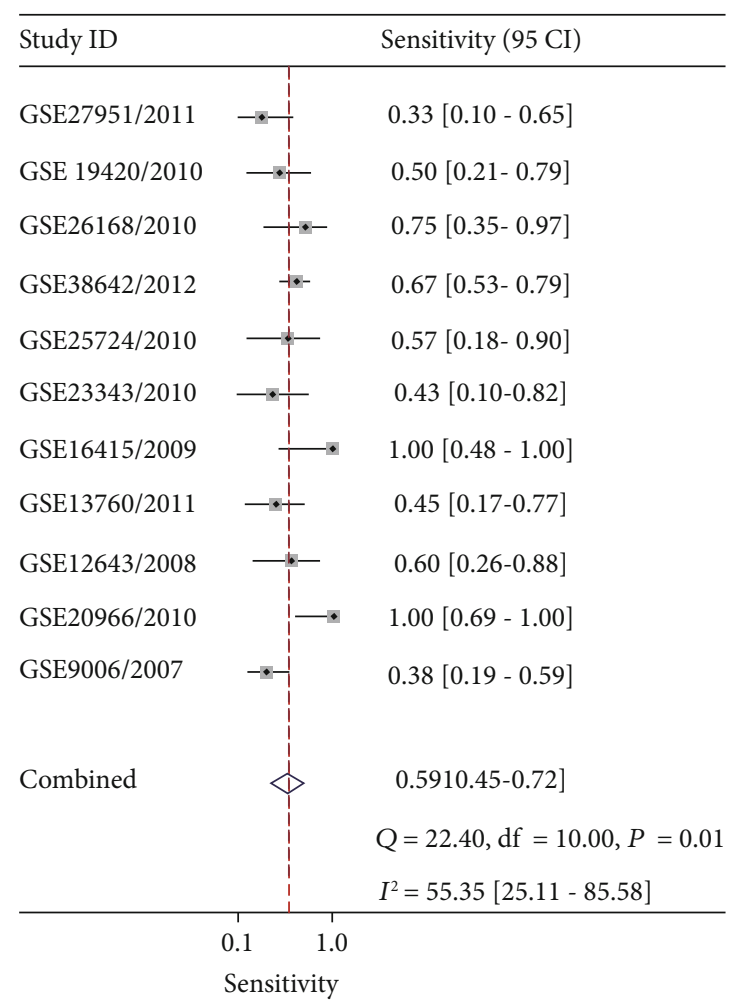

FIgURE 4: Diagnostic analysis of MIC-1 value in T2DM patients.

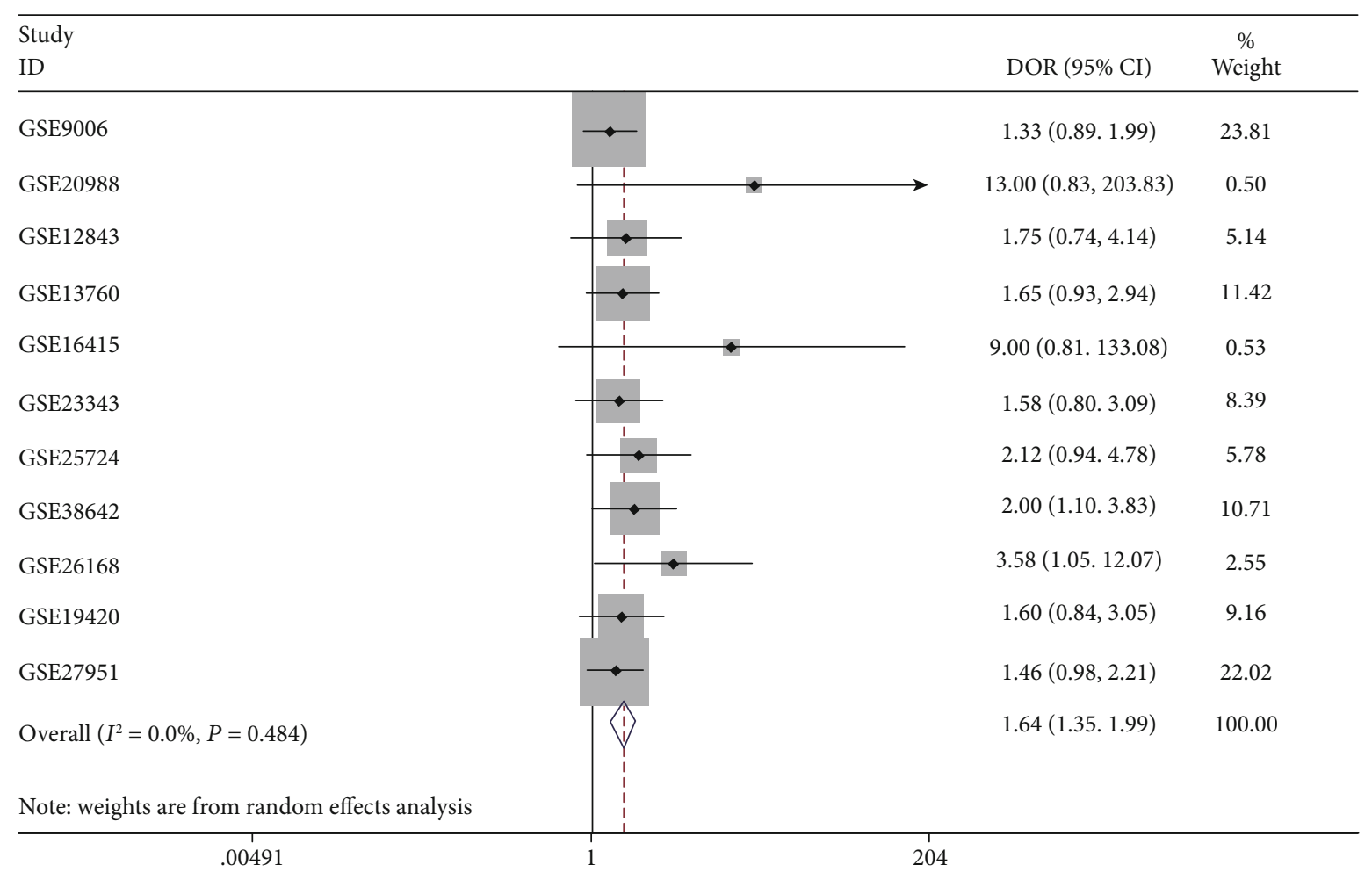

FIGURE 5: Summary receiver operating characteristic curve of $M I C-1$ value in T2DM patients. 


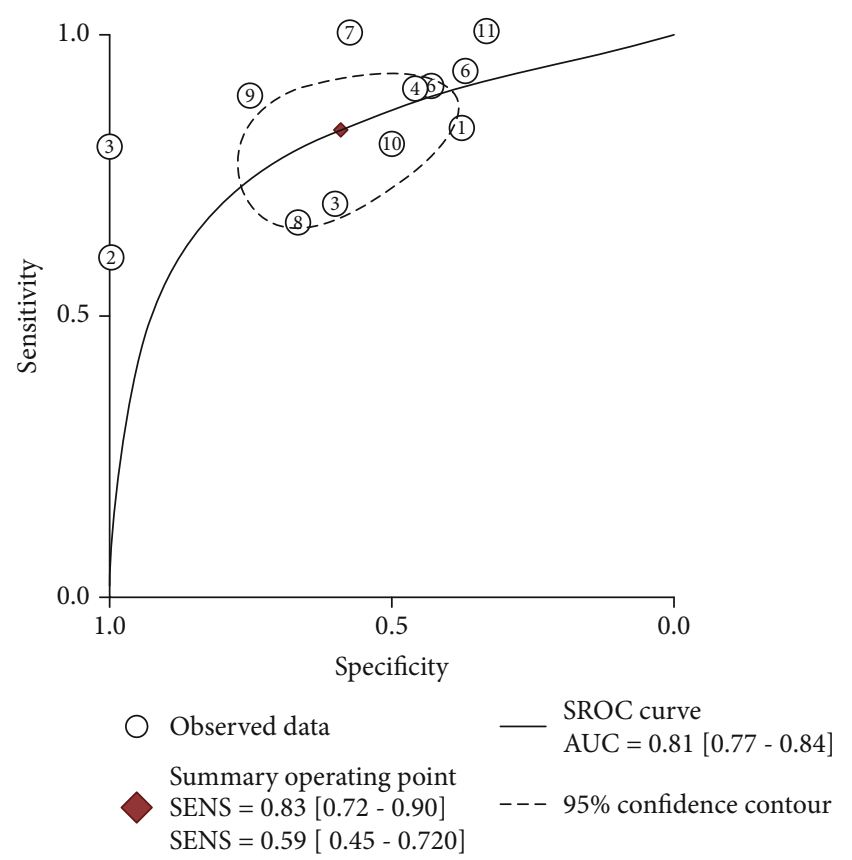

Figure 6: Sensitivity analysis of the value of MIC-1 in T2DM patients.

tended to have lower levels of MIC-1, with a SMD of -0.42 $(95 \% \mathrm{CI}=-1.09-0.25), \quad$ although it failed to reach significance (Supplementary Figure 4).

\section{Discussion}

In our report, MIC-1 value increased in different tissues of T2DM patients compared with nondiabetes people, including blood samples.

MIC-1 has been suggested to be linked with obesity and T2DM. Accumulating evidence in animals clarifies that the overexpression of MIC-1 leads to weight loss, enhanced insulin sensitivity, and higher glucose tolerance. It is assumed that MIC-1 might keep a protective factor in obese and obesity-related diseases like T2DM [25]. Firstly, previous publication has elucidated that MIC-1 acts on feeding centers in the hypothalamus and brainstem to regulate weight control [26]. Secondly, MIC-1 is a secretory product of adipocytes and plays a role in improving lipolysis which is reversely correlated with serum total cholesterol [20, 27]. Moreover, elevated MIC-1 expression could invoke improved insulin activity and mediate Th2 cytokines like IL-13 function $[14,16]$. Previous studies in human etiology of T2DM have demonstrated that high glucose would promote cellular aging and apoptosis. Acting as a protective regulator, MIC-1 expression is accordingly elevated in endothelial cells by ROS- and p53-dependent pathway [28]. MIC1 is acknowledged as an anti-inflammation cytokine and responds positively to chronic inflammatory disease like T2DM. This acknowledgment may be the reason of higher MIC-1 level in T2DM. Also, a hypothesis was proposed that MIC-1 may function as a self-guarded cytokine in T2DM accounting for reduced food intake and then weight loss. After the peak of MIC-1 expression, it is not sufficient to compensate in the long-term low-grade inflammation, leading to an unstoppable progression of weight gain and metabolism dysfunction [29]. This hypothesis is consistent with our finding that MIC-1 was found to have lower expression levels in obese patients compared with nonobese ones.

Importantly, evidence showed that the serum MIC-1 level ranks the highest in T2DM patients, intermediate in individuals with prediabetes, and lowest in patients without diabetes [20, 30-32]. Elevated serum MIC-1 expression also suggested a high risk for T2DM-related complications including cardiovascular risks $[33,34]$ and renal diseases $[35,36]$. The current study found a positive correlation between T2DM and MIC-1 level in blood samples, suggesting that MIC-1 could be a practical research target in the future.

Interestingly, an experiment in mice has reported that treating wild-type mice with both MIC-1 and liraglutide, a long-term glucagon-like peptide 1 (GLP-1) agonist, shows a synergistic impact on weight loss [18]. Progress has been made in combined utility of MIC- 1 treatment and mature clinical medicine which shows a promising route for MIC-1 as a therapy biomarker. A fair number of progress have been made in identifying GFRAL, an orphan receptor of glialderived neurotropic factor receptor $\alpha$, as a receptor for MIC-1 and its neuronal circuits in which it acts [22, 3739]. Based on this breakthrough, a class of MIC-1/GFRAL/RET-based drugs can be highly anticipated for treatment in T2DM [40].

The limitation of our study should also be of concern. First, our study estimated the contribution of numbered risk factors accounting for T2DM, such as age and BMI. Due to the limited data we obtained, we were unable to remove other influential factors such as smoking, dietary habit, and sedentary lifestyle. Second, our conclusion is summarized by a meta-analysis, causal relationship cannot be confirmed. Therefore, laboratory experiments should be performed to address the comprehensive mechanism between MIC-1 and T2DM.

\section{Conclusion}

Our study suggested that the expression level of MIC-1 was significantly higher in T2DM patients in multiple tissues including blood samples.

\section{Abbreviations}

T2DM: $\quad$ Type 2 diabetes mellitus

MIC-1: $\quad$ Macrophage-inhibiting cytokine-1

GEO: $\quad$ Gene Expression Omnibus

SMD: $\quad$ Standard mean difference

SROC: $\quad$ Summary receiver operating characteristic

GFRAL: $\quad$ Glial-derived neurotropic factor receptor $\alpha$

BMI: $\quad$ Body mass index

AUC: $\quad$ Area under the curve

MANCOVA: Multivariate analysis of covariance.

\section{Conflicts of Interest}

No potential conflict of interest was reported by the authors. 


\section{Authors' Contributions}

JY-Y has contributed to the design and concept of the manuscript. Others have contributed to the writing and revision of the paper. JN-L and YZ were responsible for the acquisition of data, analysis and interpretation of data, and manuscript drafting. HY-P, JY-L, and XX-D were responsible for the acquisition of data and analysis and interpretation of data. QZ-Y, C-Z, and JW-L were responsible for the analysis and interpretation of data and critical revision of the manuscript for intellectual content. Lu Jianan and Zhang Yue contributed equally to this study.

\section{Acknowledgments}

This work is supported by the grant from the National Key R\&D Program of China (Grant numbers 2017YFC1310700 and 2017YFC1310701) and the National Natural Science Foundation of China (Grant number 81602911).

\section{Supplementary Materials}

Supplementary Figure S.1: Begg's funnel plot for the assessment of potential publication bias in the samples. $P$ for Begg's test is 0.511 (continuity corrected). Supplementary Figure S.2: sensitivity analysis of the value of MIC-1 in T2DM patients. Supplementary Figure S.3: forest plot showing the differences in least-square means between T2DM and nonT2DM. Random model was used to test the differences. Supplementary Figure S.4: forest plot showing the SMD of MIC-1 expression between obese and nonobese T2DM patients. Fixed effect model was used in all groups. Supplementary Table S.1: characteristics of age, BMI, and MIC-1 expression levels by two groups (T2DM and nonT2DM). Supplementary Table S.2: characteristics of MIC-1 expression profiling datasets between obese and nonobese T2DM patients. (Supplementary Materials)

\section{References}

[1] N. H. Cho, J. E. Shaw, S. Karuranga et al., "IDF Diabetes Atlas: global estimates of diabetes prevalence for 2017 and projections for 2045," Diabetes Research and Clinical Practice, vol. 138, pp. 271-281, 2018.

[2] K. Ogurtsova, J. D. da Rocha Fernandes, Y. Huang et al., "IDF Diabetes Atlas: global estimates for the prevalence of diabetes for 2015 and 2040," Diabetes Research and Clinical Practice, vol. 128, pp. 40-50, 2017.

[3] G. Bruno, C. Runzo, P. Cavallo-Perin et al., "Incidence of type 1 and type 2 diabetes in adults aged 30-49 years: the population-based registry in the province of Turin, Italy," Diabetes Care, vol. 28, no. 11, pp. 2613-2619, 2005.

[4] M. J. Franz, J. L. Boucher, S. Rutten-Ramos, and J. J. VanWormer, "Lifestyle weight-loss intervention outcomes in overweight and obese adults with type 2 diabetes: a systematic review and meta-analysis of randomized clinical trials," Journal of the Academy of Nutrition and Dietetics, vol. 115, no. 9, pp. 1447-1463, 2015.
[5] S. Chatterjee, K. Khunti, and M. J. Davies, “Achieving glycaemic control with concentrated insulin in patients with type 2 diabetes," Drugs, vol. 79, no. 2, pp. 173-186, 2019.

[6] E. A. Nyenwe, C. C. Ogwo, I. Owei, J. Y. Wan, and S. DagogoJack, "Parental history of type 2 diabetes is associated with lower resting energy expenditure in normoglycemic subjects," BMJ Open Diabetes Research \& Care, vol. 6, no. 1, article e000511, 2018.

[7] M. R. Bootcov, A. R. Bauskin, S. M. Valenzuela et al., "MIC-1, a novel macrophage inhibitory cytokine, is a divergent member of the TGF-beta superfamily," Proceedings of the National Academy of Sciences of the United States of America, vol. 94, no. 21, pp. 11514-11519, 1997.

[8] Ł. A. Poniatowski, P. Wojdasiewicz, R. Gasik, and D. Szukiewicz, "Transforming growth factor Beta family: insight into the role of growth factors in regulation of fracture healing biology and potential clinical applications," Mediators of Inflammation, vol. 2015, Article ID 137823, 17 pages, 2015.

[9] A. E. Berezin, "Diabetes mellitus related biomarker: The predictive role of growth-differentiation factor-15," Diabetes \& Metabolic Syndrome: Clinical Research \& Reviews, vol. 10, no. 1, Supplement 1, pp. S154-S157, 2016.

[10] J. Koopmann, P. Buckhaults, D. A. Brown et al., "Serum macrophage inhibitory cytokine 1 as a marker of pancreatic and other periampullary cancers," Clinical Cancer Research, vol. 10, no. 7, pp. 2386-2392, 2004.

[11] L. Lind, L. Wallentin, T. Kempf et al., "Growth-differentiation factor-15 is an independent marker of cardiovascular dysfunction and disease in the elderly: results from the Prospective Investigation of the Vasculature in Uppsala Seniors (PIVUS) Study," European Heart Journal, vol. 30, no. 19, pp. 23462353, 2009.

[12] J.-. Y. Liu, X.-. X. Dong, J.-. N. Lu et al., "Utility of GDF-15 as a diagnostic biomarker in gastric cancer: an investigation combining GEO, TCGA and meta-analysis," FEBS Open Bio, vol. 9, no. 1, pp. 35-42, 2019.

[13] M. Karczewska-Kupczewska, I. Kowalska, A. Nikolajuk et al., "Hyperinsulinemia acutely increases serum macrophage inhibitory cytokin-1 concentration in anorexia nervosa and obesity," Clinical Endocrinology, vol. 76, no. 1, pp. 46-50, 2012.

[14] L. Macia, V. W. W. Tsai, A. D. Nguyen et al., "Macrophage inhibitory cytokine 1 (MIC-1/GDF15) decreases food intake, body weight and improves glucose tolerance in mice on normal \& obesogenic diets," PLoS One, vol. 7, no. 4, article e34868, 2012.

[15] H. Johnen, S. Lin, T. Kuffner et al., “Tumor-induced anorexia and weight loss are mediated by the TGF- $\beta$ superfamily cytokine MIC-1," Nature Medicine, vol. 13, no. 11, pp. 1333-1340, 2007.

[16] S. E. Lee, S. G. Kang, M. J. Choi et al., "Growth differentiation factor 15 mediates systemic glucose regulatory action of Thelper type 2 cytokines," Diabetes, vol. 66, no. 11, pp. 27742788, 2017.

[17] H. K. Chung, D. Ryu, K. S. Kim et al., "Growth differentiation factor 15 is a myomitokine governing systemic energy homeostasis," The Journal of Cell Biology, vol. 216, no. 1, pp. 149-165, 2017.

[18] H. Frikke-Schmidt, K. Hultman, J. W. Galaske, S. B. Jørgensen, M. G. Myers Jr., and R. J. Seeley, "GDF15 acts synergistically with liraglutide but is not necessary for the weight loss induced by bariatric surgery in mice," Molecular Metabolism, vol. 21, pp. 13-21, 2019. 
[19] S. N. Breit, H. Johnen, A. D. Cook et al., "The TGF- $\beta$ superfamily cytokine, MIC-1/GDF15: a pleotrophic cytokine with roles in inflammation, cancer and metabolism," Growth Factors, vol. 29, no. 5, pp. 187-195, 2011.

[20] Q. Ding, T. Mracek, P. Gonzalez-Muniesa et al., "Identification of macrophage inhibitory cytokine- 1 in adipose tissue and its secretion as an adipokine by human adipocytes," Endocrinology, vol. 150, no. 4, pp. 1688-1696, 2009.

[21] N. Zamani and C. W. Brown, "Emerging roles for the transforming growth factor-\{beta\} superfamily in regulating adiposity and energy expenditure," Endocrine Reviews, vol. 32, no. 3, pp. 387-403, 2011.

[22] J.-Y. Hsu, S. Crawley, M. Chen et al., "Non-homeostatic body weight regulation through a brainstem-restricted receptor for GDF15," Nature, vol. 550, no. 7675, pp. 255-259, 2017.

[23] G. F. Cahill Jr., "Medical associations and the pace of change," The Western Journal of Medicine, vol. 142, no. 2, pp. 249-250, 1985.

[24] A. Engin, "The definition and prevalence of obesity and metabolic syndrome," in Obesity and Lipotoxicity, A. B. Engin and A. Engin, Eds., pp. 1-17, Springer International Publishing, Cham, 2017.

[25] M. Tang, M. Luo, W. Lu et al., "Serum growth differentiation factor 15 is associated with glucose metabolism in the third trimester in Chinese pregnant women," Diabetes Research and Clinical Practice, vol. 156, article 107823, 2019.

[26] V. W. W. Tsai, S. Lin, D. A. Brown, A. Salis, and S. N. Breit, "Anorexia-cachexia and obesity treatment may be two sides of the same coin: role of the TGF-b superfamily cytokine MIC-1/GDF15," International Journal of Obesity, vol. 40, no. 2, pp. 193-197, 2016.

[27] A. E. Achari and S. K. Jain, "Adiponectin, a therapeutic target for obesity, diabetes, and endothelial dysfunction," International Journal of Molecular Sciences, vol. 18, no. 6, p. 1321, 2017.

[28] J. Li, L. Yang, W. Qin, G. Zhang, J. Yuan, and F. Wang, “Adaptive induction of growth differentiation factor 15 attenuates endothelial cell apoptosis in response to high glucose stimulus," PLoS One, vol. 8, no. 6, article e65549, 2013.

[29] X. Bao, Y. Borné, I. F. Muhammad et al., "Growth differentiation factor 15 is positively associated with incidence of diabetes mellitus: the Malmö Diet and Cancer-Cardiovascular Cohort," Diabetologia, vol. 62, no. 1, pp. 78-86, 2019.

[30] J. H. Hong, H. K. Chung, H. Y. Park et al., "GDF15 is a novel biomarker for impaired fasting glucose," Diabetes \& Metabolism Journal, vol. 38, no. 6, pp. 472-479, 2014.

[31] T. Kempf, A. Guba-Quint, J. Torgerson et al., "Growth differentiation factor 15 predicts future insulin resistance and impaired glucose control in obese nondiabetic individuals: results from the XENDOS trial," European Journal of Endocrinology, vol. 167, no. 5, pp. 671-678, 2012.

[32] I. Dostálová, T. Roubíček, M. Bártlová et al., "Increased serum concentrations of macrophage inhibitory cytokine-1 in patients with obesity and type 2 diabetes mellitus: the influence of very low calorie diet," European Journal of Endocrinology, vol. 161, no. 3, pp. 397-404, 2009.

[33] A. Dominguez-Rodriguez, P. Abreu-Gonzalez, and P. Avanzas, "Usefulness of growth differentiation factor- 15 levels to predict diabetic cardiomyopathy in asymptomatic patients with type 2 diabetes mellitus," The American Journal of Cardiology, vol. 114, no. 6, pp. 890-894, 2014.
[34] M. Y. Shin, J. M. Kim, Y. E. Kang et al., “Association between growth differentiation factor 15 (GDF15) and cardiovascular risk in patients with newly diagnosed type 2 diabetes mellitus," Journal of Korean Medical Science, vol. 31, no. 9, pp. 14131418, 2016.

[35] J. H. Hong, Y.-K. Choi, B.-K. Min et al., "Relationship between hepcidin and GDF15 in anemic patients with type 2 diabetes without overt renal impairment," Diabetes Research and Clinical Practice, vol. 109, no. 1, pp. 64-70, 2015.

[36] M. Mazagova, H. Buikema, A. van Buiten et al., "Genetic deletion of growth differentiation factor 15 augments renal damage in both type 1 and type 2 models of diabetes," American Journal of Physiology-Renal Physiology, vol. 305, no. 9, pp. F1249-F1264, 2013.

[37] L. Yang, C. C. Chang, Z. Sun et al., "GFRAL is the receptor for GDF15 and is required for the anti-obesity effects of the ligand," Nature Medicine, vol. 23, no. 10, pp. 1158-1166, 2017.

[38] S. E. Mullican, X. Lin-Schmidt, C. N. Chin et al., "GFRAL is the receptor for GDF15 and the ligand promotes weight loss in mice and nonhuman primates," Nature Medicine, vol. 23, no. 10, pp. 1150-1157, 2017.

[39] P. J. Emmerson, F. Wang, Y. du et al., "The metabolic effects of GDF15 are mediated by the orphan receptor GFRAL," Nature Medicine, vol. 23, no. 10, pp. 1215-1219, 2017.

[40] F. Li, X. Ruan, and L. Min, "Targeting both sides of the GDF15-GFRAL-RET receptor complex: a new approach to achieve body weight homeostasis," Genes \& Diseases, vol. 4, no. 4, pp. 183-184, 2017.

[41] M. Carstensen, C. Herder, E. J. Brunner et al., "Macrophage inhibitory cytokine- 1 is increased in individuals before type 2 diabetes diagnosis but is not an independent predictor of type 2 diabetes: the Whitehall II study," European Journal of Endocrinology, vol. 162, no. 5, pp. 913-917, 2010. 


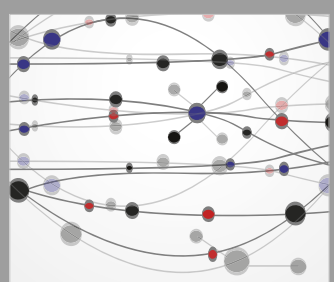

The Scientific World Journal
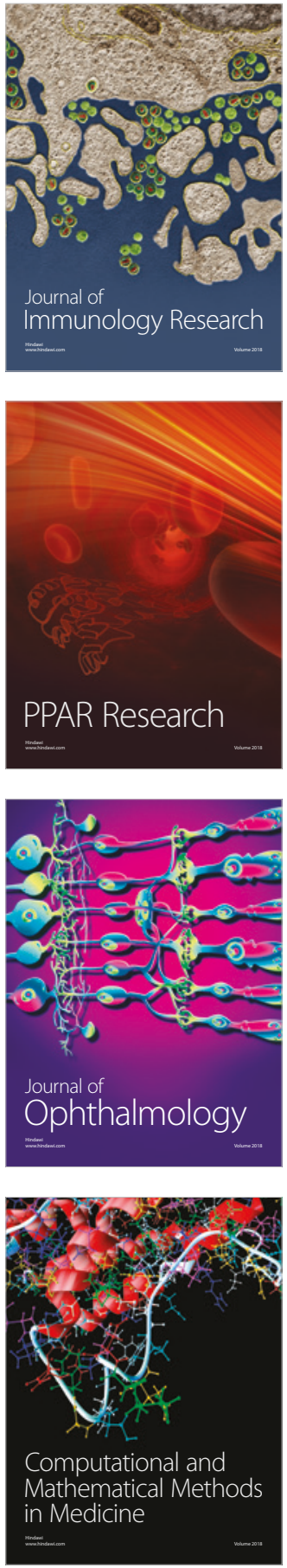

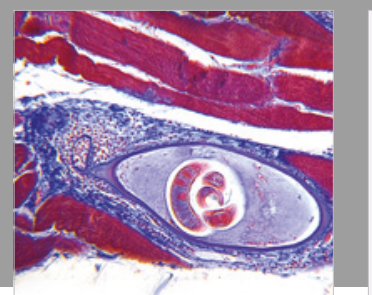

Gastroenterology Research and Practice

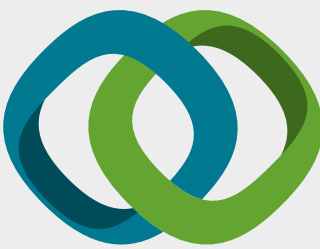

\section{Hindawi}

Submit your manuscripts at

www.hindawi.com
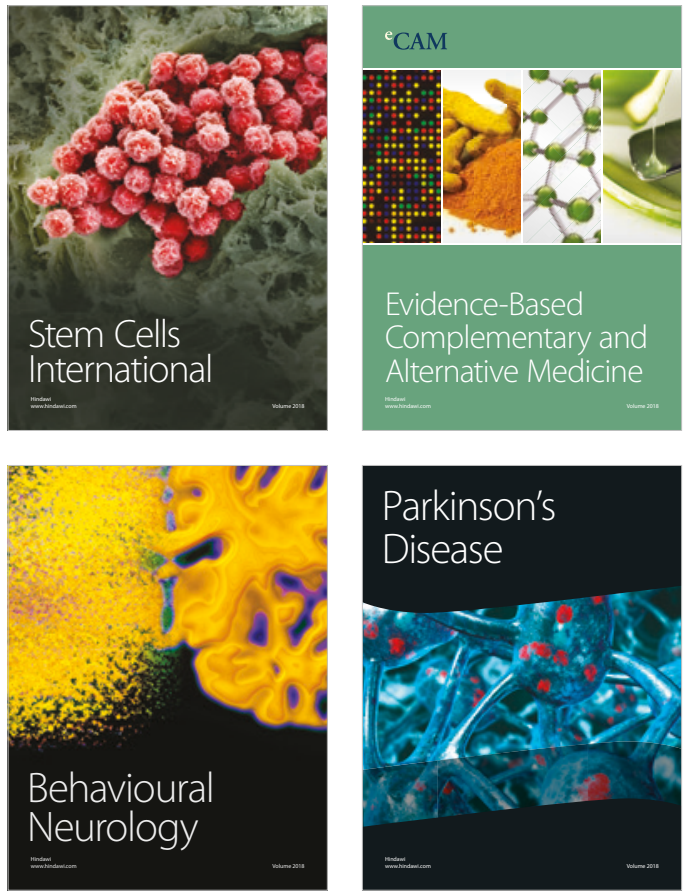

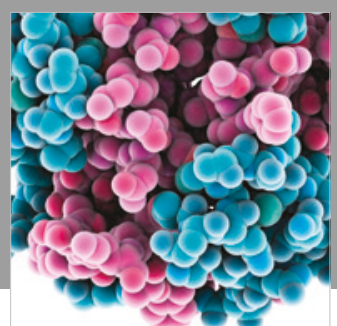

ournal of

Diabetes Research

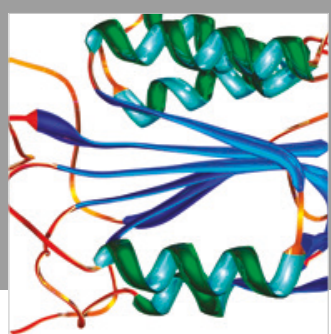

Disease Markers
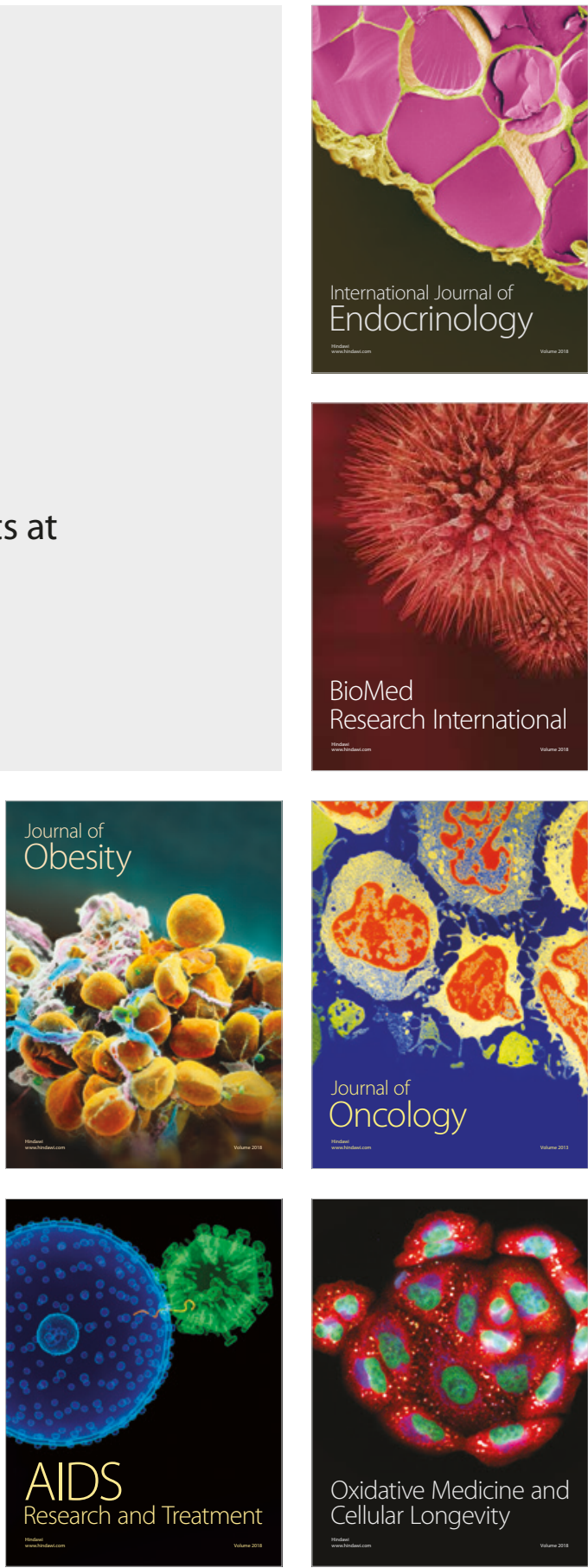